\title{
Numerical Simulations of Flow in Electrostatic Precipitators
}

\author{
Richard Matas ${ }^{1, *}$, Martin Novák ${ }^{2}$, and Jindřich Kňourek ${ }^{1}$ \\ ${ }^{1}$ Modelling and measurement of interactions in technical systems, New Technologies - Research \\ Centre, University of West Bohemia, Univerzitní 2732/8, 30100 Pilsen, Czech Republic \\ ${ }^{2}$ Department of Power System Engineering, Faculty of Mechanical Engineering, University of West \\ Bohemia, Univerzitní 2762/22, 30100 Pilsen, Czech Republic
}

\begin{abstract}
Electrostatic precipitators are widely used in power generation and industry to separate solid particles from flue gases. They need relatively homogeneous distribution of the flue gas inside otherwise the required efficiency cannot be ensured. The paper shows how to approach some of possible troubles with distribution of the stream inside of the filters caused by unsuitable design of ducts in front of the device or by inlet parts of filters. Three filters in operation are presented as examples which seem to be similar, but their behaviour is different. CFD simulations partialy validated by measurement were used to evaluate the existing flow situation. Design changes were proposed and further investigated to improve the flows in the precipitator.
\end{abstract}

\section{Introduction}

An electrostatic precipitator (ESP) is a device which is widely used to remove particles from a flue gas. These devices use electrostatic charging to achieve the desired effect. The flue gas goes through a filter chamber, the particles in it are charged and these ionized particles are diverted to the ground plates where they are collected. This is a simplified process of filtration and it is expected that almost all particles are filtered from the flue gas [1]. To achieve the highest filter efficiency, it is necessary to keep proper flue gas distribution along the whole length of the filter chambers (and the collecting plates) because if some parts of the filters are overloaded and some of them are not used this causes higher emissions, which is undesirable.

The experimental measurement of velocity profiles in an ESP is possible but expensive and complicated and it is limited only to the detection of problems or for the attestation of the finished device. CFD simulations can help to create the required profiles in the ESP or to improve an unsatisfactory profile. CFD simulations can significantly contribute to the development process of a new or reconstructed device.

An ESP is a very complex device containing a lot of parts which are important because each could influence the flow distribution inside the filter. It is necessary to use simplified geometry for CFD simulations, which includes removing bolts and small plates, etc.

\footnotetext{
*Corresponding author: mata@ntc.zcu.cz
} 
Some authors use higher levels of simplifications such as [2], [3], [4] where they simulate half the filter because they are usually symmetrical. Some authors such as [3] even neglect the inlet duct which has a huge influence on the flow distribution in the filter, as in [5] and [2]. Some authors, such as [6] separate simulations into two different parts. The first is a simulation of the duct and the results from it are used as the input to the second simulation of the filter. It is therefore also necessary to design the duct in front of the filter well, as it has a significant effect on the flow situation inside the filter, as evidenced by, for example, [7].

This paper focuses on complex CFD simulation of flow in ESPs. Three different filters are described as case studies. Each filter needs a special approach because even though the main filtration chambers are similar, the ducts and inlet parts in front of them are always different. All these filters are on site and in operation.

The CFD simulations were done with ANSYS Fluent and the main reasons for conducting these calculations were to find out the current situation of the gas (and in some cases also dust) distribution in the filters and possible feasibility studies of modifications for improving efficiency of the ESPs.

\section{Electrostatic precipitator no. 1}

This ESP was simulated to find the strengths and weaknesses of the design and make possible changes to increase the filtration process. The current ESP design is sufficient at the moment, but new regulations might come out making it necessary to make fast improvements to it.

The geometry of the filter is shown in Figure 1 on the left. The filter was fully modelled with the inlet and outlet ducts. Some simplifications were made, such as: neglecting bolts, curvature of collecting electrodes, etc. The perforated plate with flaps was modelled according to design specifications because this is a crucial part of the geometry and it is very important to consider the effect of this plate. The plate is shown in Figure 1 on the right.

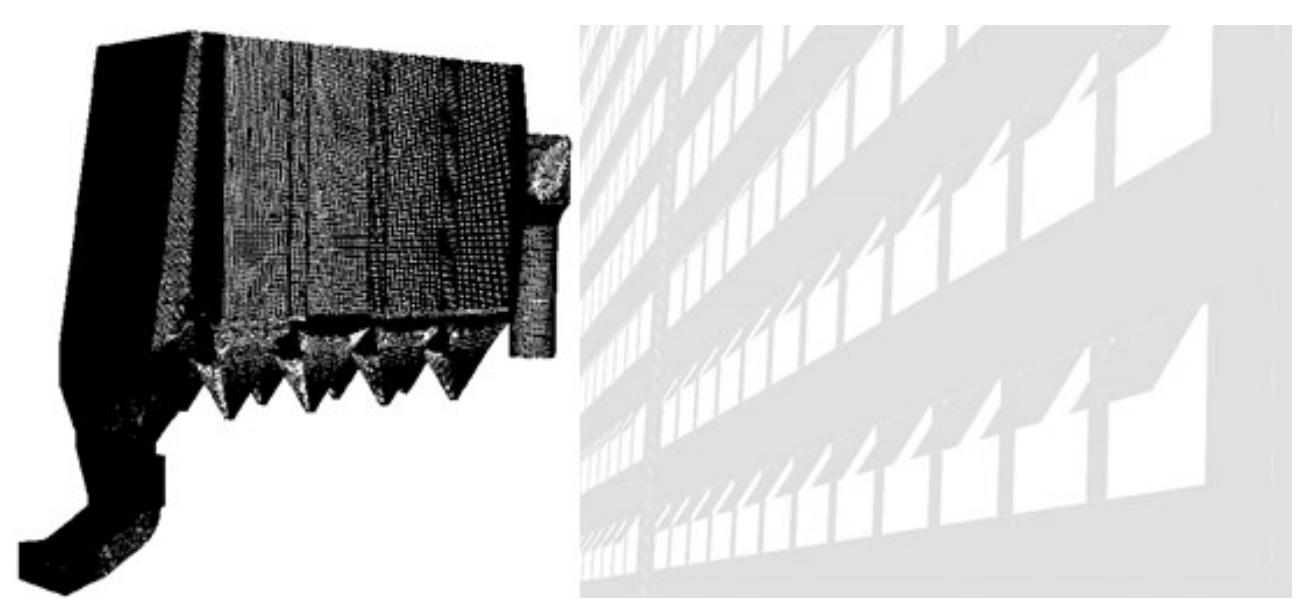

Fig. 1. Geometry of the filter (left), geometry of perforated plate with flaps (right).

The simulations were done as a simple fluid flow without a discrete phase. The mass flow rate through the filter is $147[\mathrm{~kg} / \mathrm{s}]$, so the inlet velocity is $12[\mathrm{~m} / \mathrm{s}]$. The turbulent model used in the simulations was SST k- $\omega$ and all simulations were done as steady state. 
Simulations of the basic geometry were done and evaluated. These results were used to identify parts of the geometry where improvements could be made. The improvements were made in three possible simple variants which are shown in Figure 2.
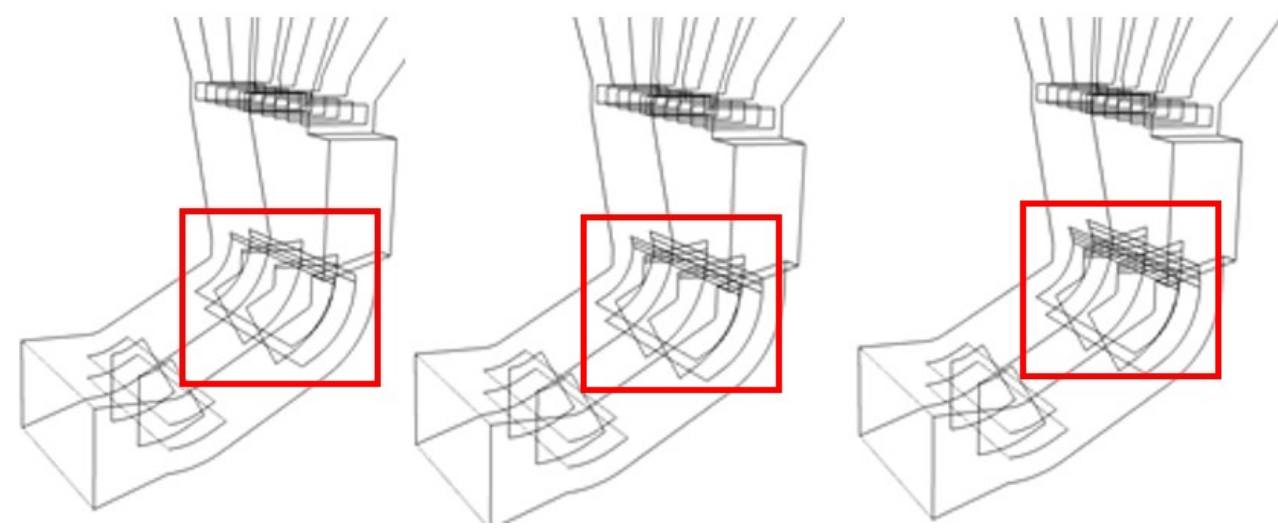

Fig. 2. Geometry modification of ESP 1, mark 1 (left), mark 2 (middle) and mark 3 (right).

The variants are based on the initial one. The inserts in the inlet duct are almost the same. The changes are made only in the second elbow on the inserts which are highlighted in Figure 2 with red rectangles. The inserts are extended in the flow direction and rotated at an angle. The angle in mark 1 is $5^{\circ}$, mark 2 is longer than mark 1 and the angle is $10^{\circ}$, mark 3 has the biggest extension of inserts and the angle is $15^{\circ}$.

Before evaluation, it is necessary to suggest the right places to observe the changes of the stream in the ESP filter. It is expected that the flow might be focused only in one part of the filter which would overload this part, leaving some parts of the filter almost idle. It is necessary to provide proper distribution of the fluid flow in the inlet chamber of the filter and it is important to check the distribution inside the filter. To check these phenomena three postprocessing planes are suggested, as shown in Figure 3.
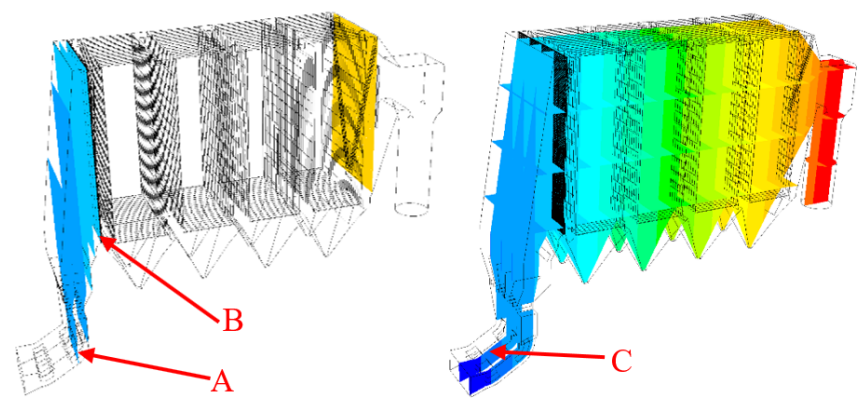

Fig. 3. Evaluation planes.

The first evaluation of the initial variant and designed marks is shown in Figure 4. These are velocity magnitudes $[\mathrm{m} / \mathrm{s}]$. The initial variant shows that the stream here is pushed to the right side of the filter. Mark 1 distributes the stream in a similar way to the initial variant. Marks 2 and 3 slow the stream and distribute it to the middle part of the inlet chamber of the filter. 

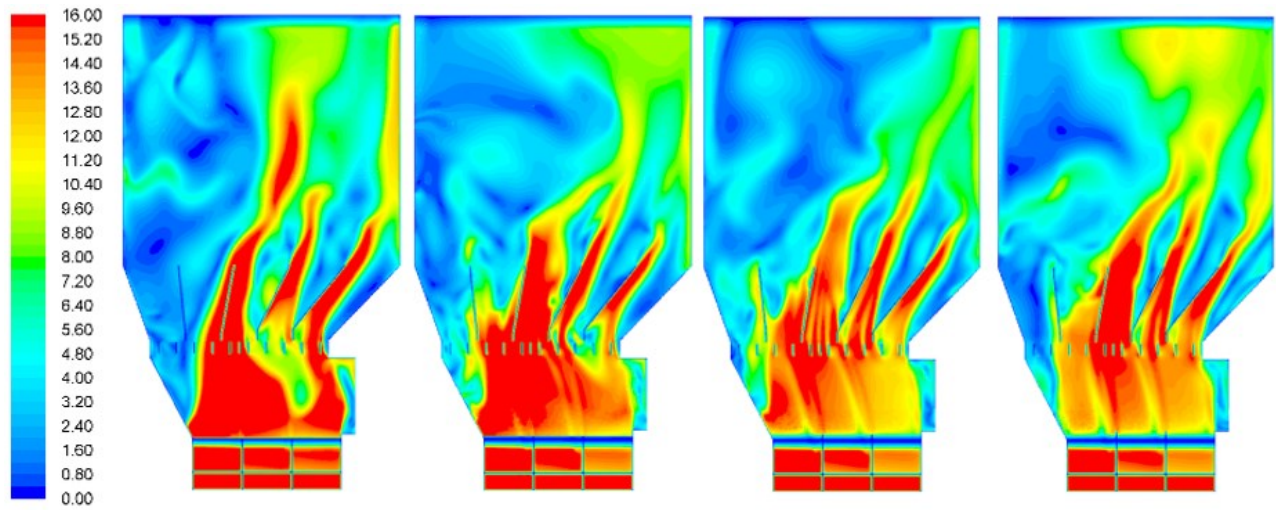

Fig. 4. Velocity magnitude $[\mathrm{m} / \mathrm{s}]$ contours on initial variant (first from left), mark 1 (second from left), mark 2 (third from left) and mark 3 (fourth from left) on plane A.

The next set of contours on plane B is shown in Figure 5. Plane B is in front of the filtration chamber of the filter, so this is a crucial part where the stream should be well distributed because it affects the flow in the whole filter. The initial variant shows that there are high velocities in the lower part of the filter, so it is necessary to distribute it better. The results from marks 1, 2 and 3 show that the modifications can slow down the flow. The lowest velocities are reached in marks 2 and 3.
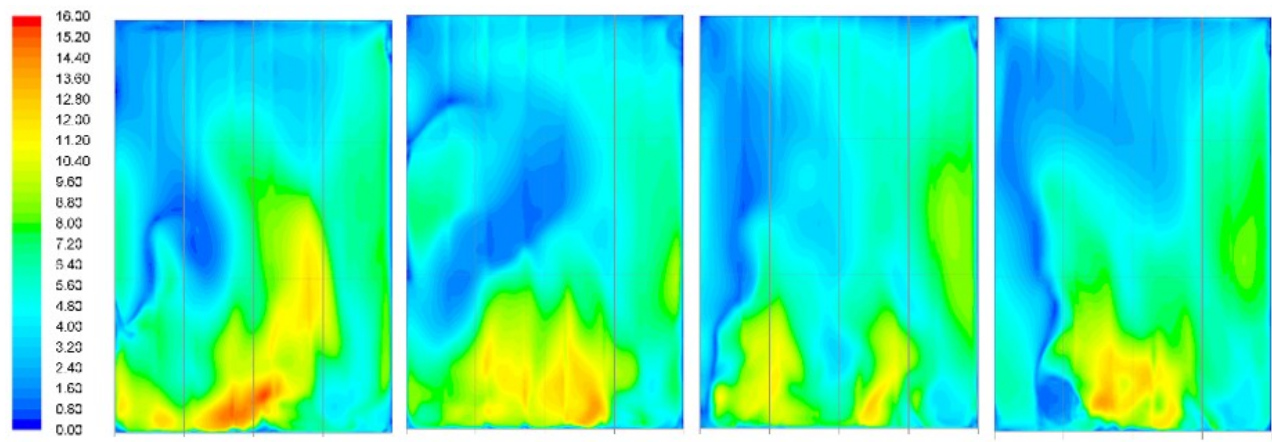

Fig. 5. Velocity magnitude $[\mathrm{m} / \mathrm{s}]$ contours on basic variant (first from left), mark 1 (second from left), mark 2 (third from left) and mark 3 (fourth from left) on plane B.

The last set of contours is displayed in Figure 6. These contours show the stream along the whole ESP filter. The blue rectangle in the figures is caused by the location of plane C which interferes with the first collecting electrode. The figure shows that the initial variant before the plate with flaps has high velocities in the lower part of the inlet chamber but in the filtration chamber the stream is not equally distributed and it is moved to the upper part. Mark 2 magnifies the effect of the initial variant, so the filtration chamber is overloaded in the top and the middle, and the lower part is under-loaded. Mark 3 slightly improves the problem with overloading of the upper filtration part. Mark 1 has the best results from this point of view because it distributes the flow almost equally along the whole filtration chamber from the bottom to the top, but mark 2 is the best compromise from the overall point of view. 


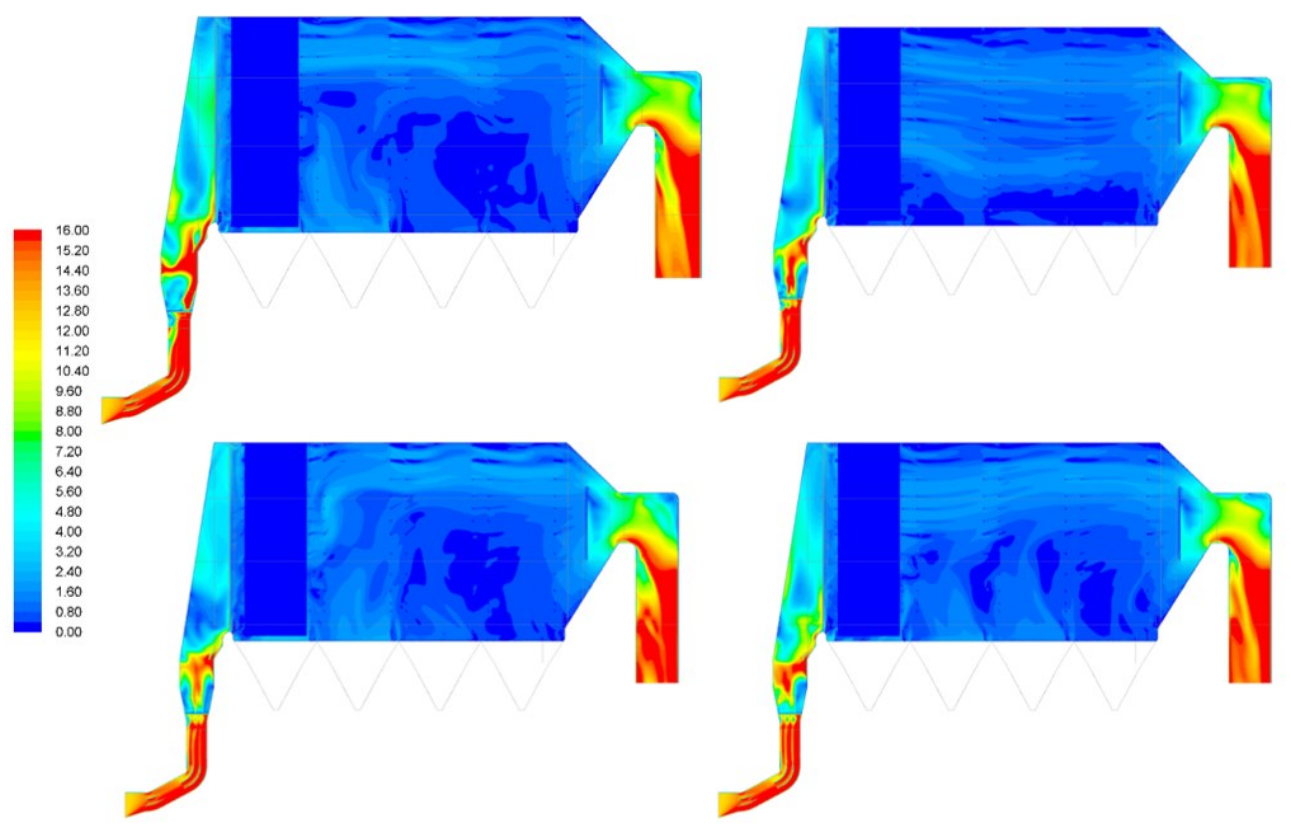

Fig. 6. Velocity magnitude $[\mathrm{m} / \mathrm{s}]$ contours on initial variant (upper left), mark 1 (upper right), mark 2 (bottom left) and mark 3 (bottom right) on plane $\mathrm{C}$.

These results also show that a small change in the inlet duct has a significant influence on the whole system, so it is important to make simulations and optimise the whole inlet duct, which is often neglected.

\section{Electrostatic precipitator no. 2}

This ESP could be called 'the smaller one' because it only has three sections and only one filtration chamber. Although it is relatively small it is necessary to guarantee its proper functioning. The reason for these simulations was to reduce some weaknesses of the design. The efficiency of the device has been not satisfactory and quite massive impurities have also been observed. This causes the flow of the flue gas to not go properly through the ESP and this escalates to the deterioration of filtration efficiency and the impurities must be cleaned before the system is reactivated.

Figure 7 shows the geometry of ESP 2 from the left. This filter has only one distribution plate and one louver plate which should distribute the flow homogenously to the filtration chamber. On the right is the computational mesh. The whole filter was modelled with a relatively long inlet duct but with only a short outlet duct which does not have any significant influence on the flow situation inside the filter. 


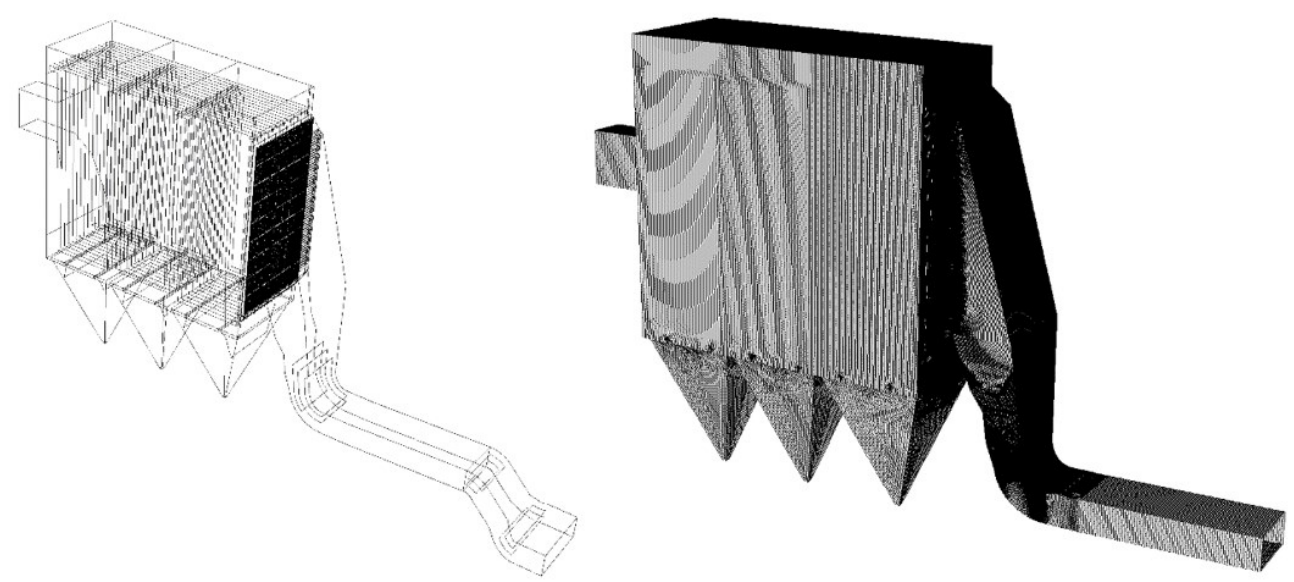

Fig. 7. Geometry of ESP 2 (left), mesh of ESP 2 (right).

The distribution perforated plate is fully modelled and is shown in Figure 8. The design of this plate is complicated because there are many sections and there are a lot of different types of holes in it. To properly simulate the whole filter, it was necessary to have the same design as it really is in the power plant - there could not be any simplifications.

The values for the numerical model were based on the experimental measurement realized with the air thus with higher density then the regime with the flue gas but from the results of previous projects showed that this difference does not affect the flow in the ESP considerably. The mass flow rate through the filter was also set as $64.4[\mathrm{~kg} / \mathrm{s}]$, the density $1.13\left[\mathrm{~kg} / \mathrm{m}^{3}\right]$ and the model settings were the same as for the ESP 1 .

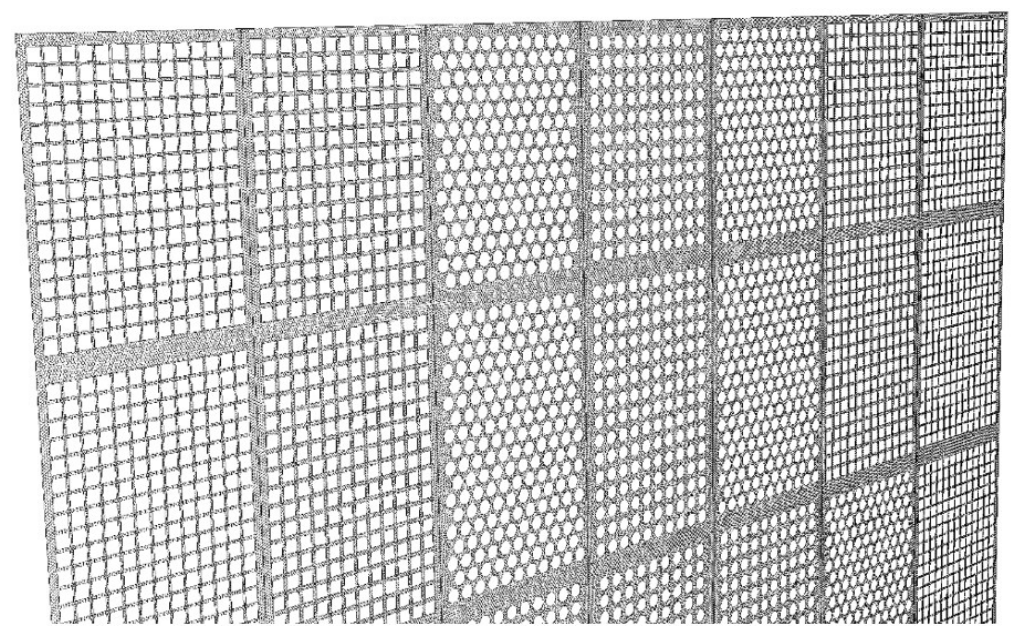

Fig. 8. Mesh of the distribution perforated plate.

One of the biggest drawbacks of this filter was the design of louver plate shown in Figure 9. In the figure you can see the geometry of the louver plate which is basically made of many L-profiles. The left side of the figure contains pathlines and the right side represents vectors coloured by velocity magnitude. Vortexes are shown behind the louvers which can cause solid pollutants to settle. This phenomenon can be so significant during a longer operation time that the pollutants can block whole gaps between two louvers. 
The shape and placement of the louver plate was evaluated as inappropriate, so this was one of the parts which was further modified.
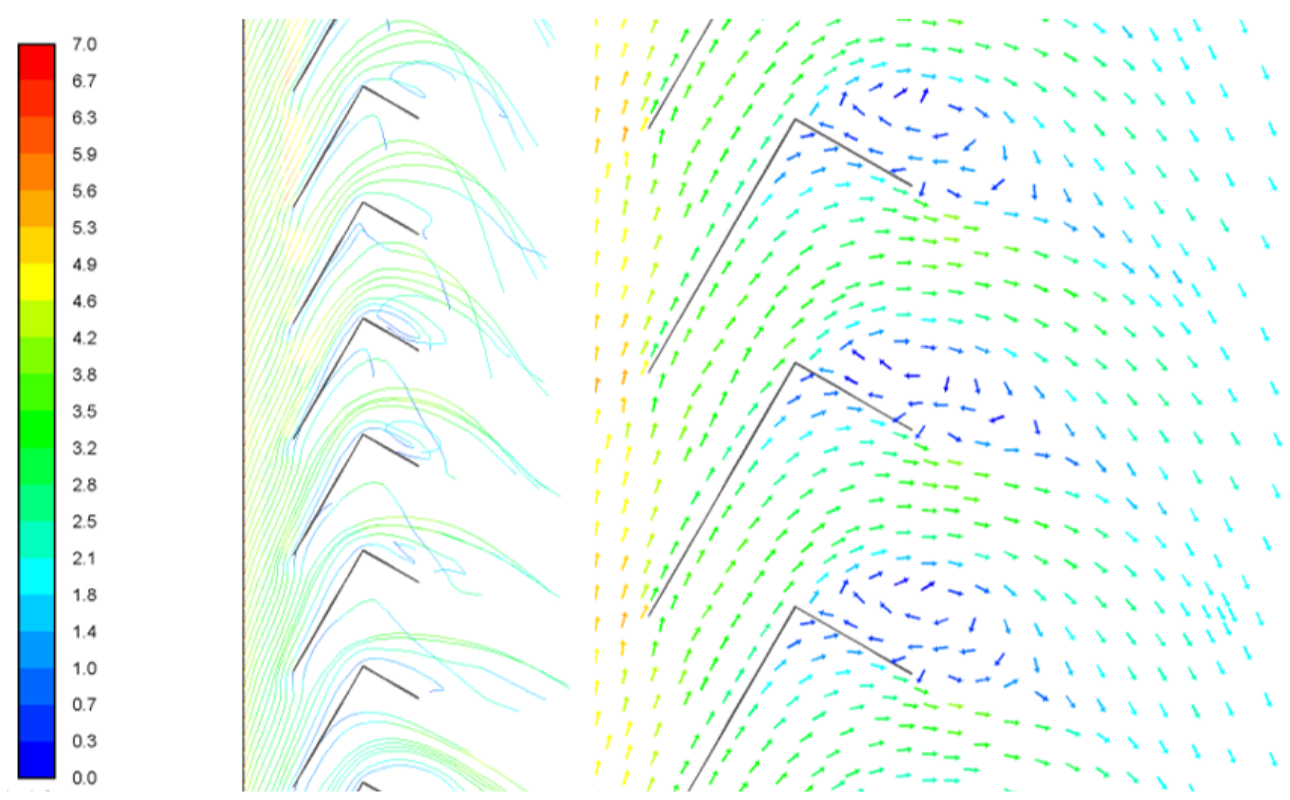

Fig. 9. Pathlines coloured by velocity magnitude $[\mathrm{m} / \mathrm{s}]$ (left), vectors coloured by velocity magnitude $[\mathrm{m} / \mathrm{s}]$ (right).

A number of different numerical simulations were made and some of them showed interesting results. For example, simulations of this filter without the inlet duct were done, meaning that the inlet profile was homogenous. The results from this simulation show that the this filter was designed without considering the inlet duct, as the louver plate worked well in this case, but in real operating conditions it resulted in ineffective filtration.

The experimental values were measured on the real filter to partially validate the CFD simulations. The experimental results are displayed in Figure 10 on the left. The results from CFD simulation in the same location are shown on the right. The results are velocities in $\mathrm{x}$ direction which is the direction of the flow through the filter chambers. The results from CFD simulation and the experiment have a similar character but the maximum values are shifted. This might be caused by the experimental approach (the measurement of relatively low velocities in large spaces has big uncertainties) and also by the simplifications of the real ESP geometry in the CFD model. 

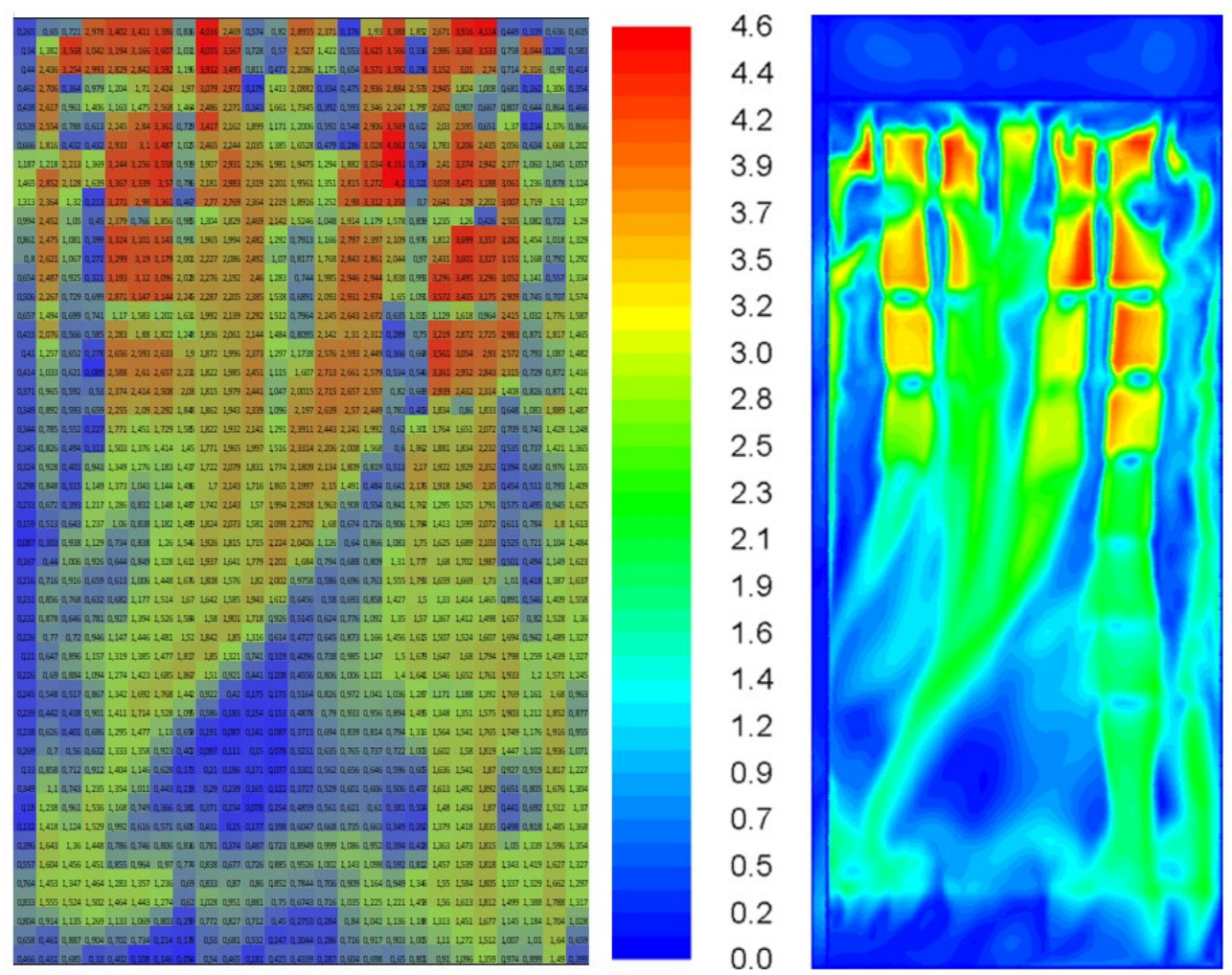

Fig. 10. Experimental data of velocities $[\mathrm{m} / \mathrm{s}]$ in $\mathrm{x}$ direction (left), contours of velocities $[\mathrm{m} / \mathrm{s}]$ in $\mathrm{x}$ direction from CFD simulation (right).

Many simulations were performed with various modifications of the geometry, but the results were often unsatisfactory. It was found that small changes inside the duct or in the inlet chamber of the filter are not sufficient to improve the overall flow distribution through the filter, and it was virtually impossible to eliminate the vortexes behind the louvers.

To guarantee the proper flow distribution inside the filter chamber and to eliminate the vortexes behind the louvers it was necessary to basically create a new shape of the inlet chamber and their inserts. Several dozen simulations were done and other development modifications to the design were made, but the description of this process is not a part of this publication. The final design is shown in Figure 11. The original perforated plate remains but there is a new one in front of it. The new perforated plate is angled and is shown in purple in Figure 11. The louver plate was modified, and its shape is not constant. The individual louvers have different shapes and geometries according to the requirements of the non-homogenous inlet stream. 


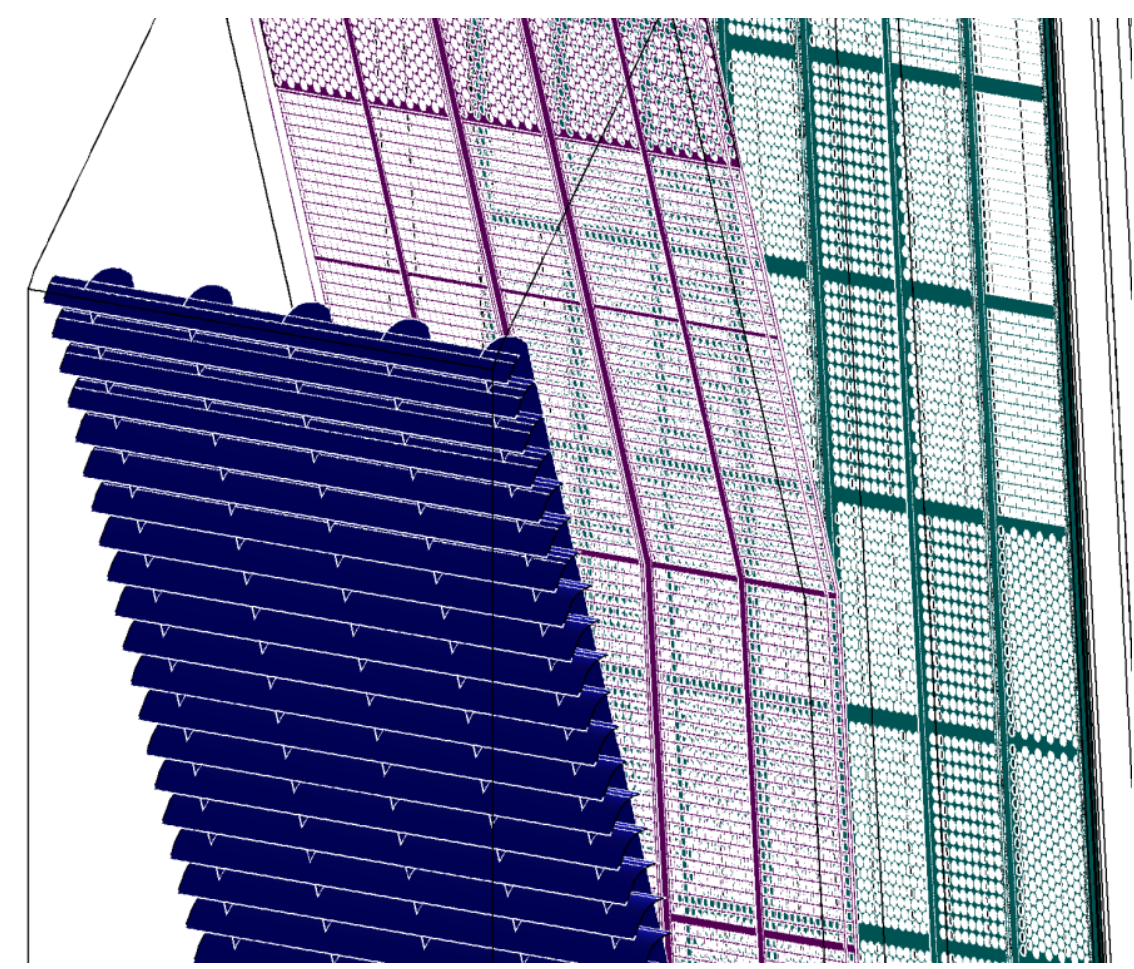

Fig. 11. Final design modification of the inlet chamber

Figure 12 compares the initial variant and the new final design - the original geometry is on the left and the new one on the right. Velocity magnitude contours $[\mathrm{m} / \mathrm{s}]$ are shown on the inlet to the filter chamber. The original geometry generates high values of local velocities and the velocity distribution is not homogenous. The new design lowers the velocities and the distribution is more uniform even though the upper part has higher velocities than the lower one, but generally the flow situation is highly improved and the backflow is eliminated. 

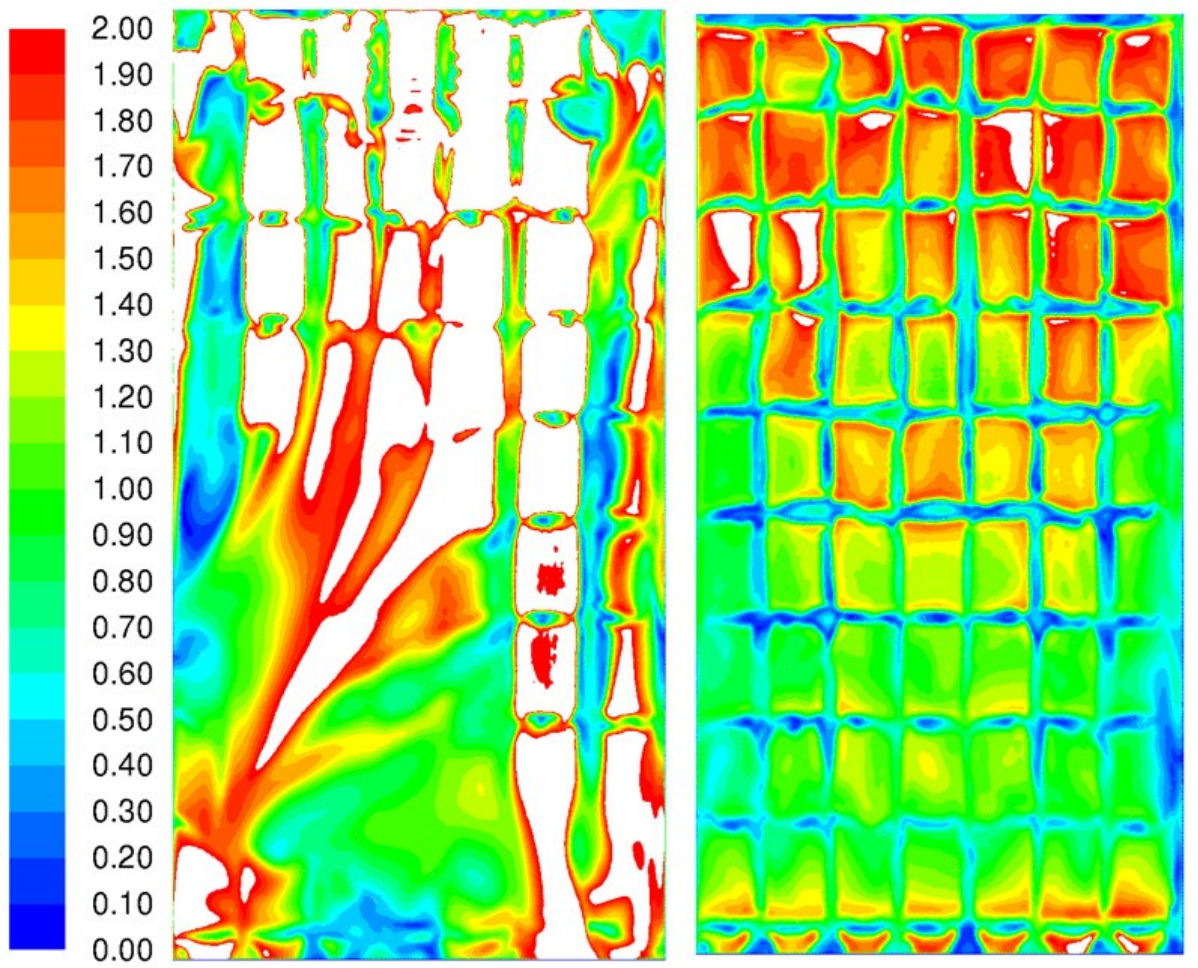

Fig. 12. Velocity magnitude countours on the inlet to the filtration chamber $[\mathrm{m} / \mathrm{s}]$ of the initial geometry (left) and the new geometry (right).

Velocity magnitude contours $[\mathrm{m} / \mathrm{s}]$ are also displayed in Figure 13 . On the left is the original variant and on the right is the newly designed one. The flow situation is highly improved and stabilized and the distribution of the flow is more uniform. This surface is a cross section exactly in the middle of the filter and the other places are similar. 

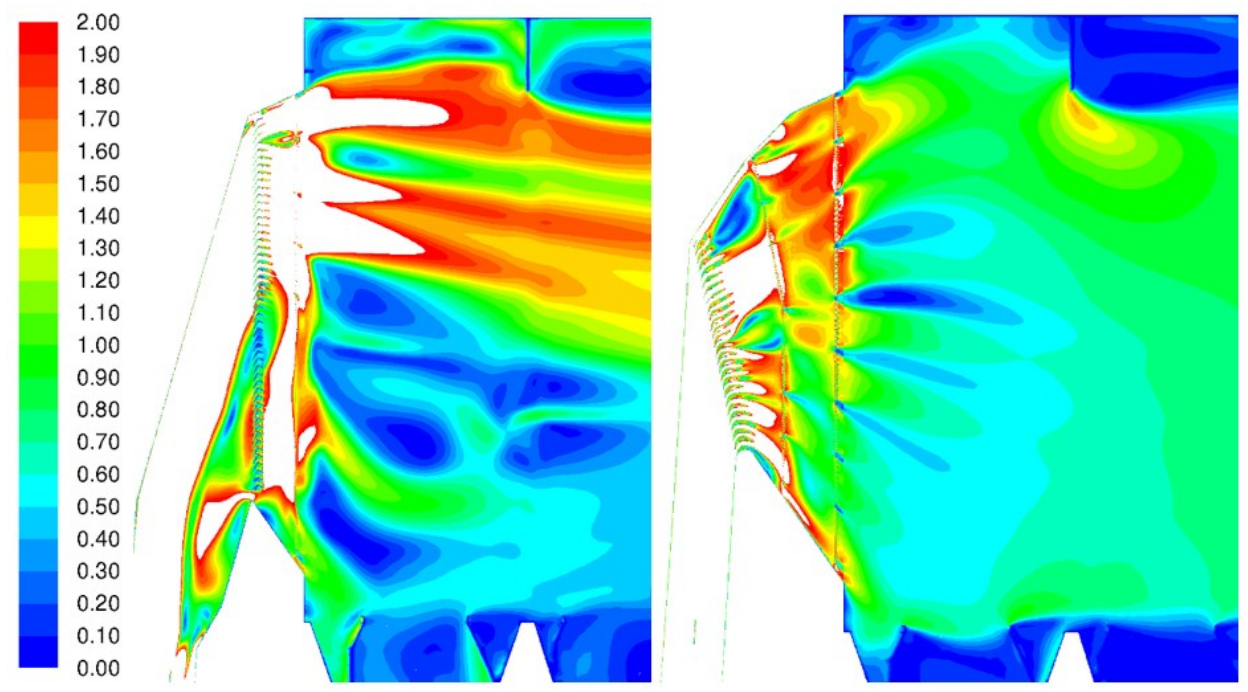

Fig. 13. Velocity magnitude contours $[\mathrm{m} / \mathrm{s}]$ in the middle cross section of the initial geometry (left) and the new geometry (right).

The optimization was done satisfactorily as the results above show and also, there were further improvements made to the final variant because it was necessary to reduce the weight and cost, but this had no effect on the results of the flow fields.

\section{Electrostatic precipitator no. 3}

The third case study, ESP 3, is a relatively unusual type of precipitator, because the inlet duct is oblique. This meant that it was not possible to use a standardized design and it was necessary to make a special design for the inlet part of the filter. There were strict geometric restrictions for the inlet duct because no changes could be made to its surroundings.

The simulations were done with only half the filter because the inlet duct and the filter are symmetrical, so the simplification should not cause any significant loss of information. The model is shown in Figure 14.

The inlet is filled with perforated plates and a vane wall. There are three perforated plates with three different types of holes in them; there is always the same raster which is $50 \mathrm{~mm}$. The first plate has square holes 39x39 mm, shown in green. The second plate has two parts, the first part is green, and the square holes are $39 \times 39 \mathrm{~mm}$. The yellow part of the second plate has circular holes with a diameter of $37 \mathrm{~mm}$. The last, third plate has three different sections and therefore three colours. The green part has 39x39 mm square holes, the yellow section has circular holes with a diameter of $37 \mathrm{~mm}$. The last, red part also has circular holes with a diameter of $33 \mathrm{~mm}$.

The direct modelling of the plates was too time consuming for pre-processing and CFD simulations. It is possible to use a specialized User Defined Function (UDF) to create a similar effect to the perforated plate. The UDFs were developed in a separate research project, tested on many CFD models of ESPs and work fine. These UDFs can significantly decrease the number of cells in the mesh and create the mesh easily. The UDF creates a pressure drop which is a function of the hole raster and the angle of attack of the stream. 


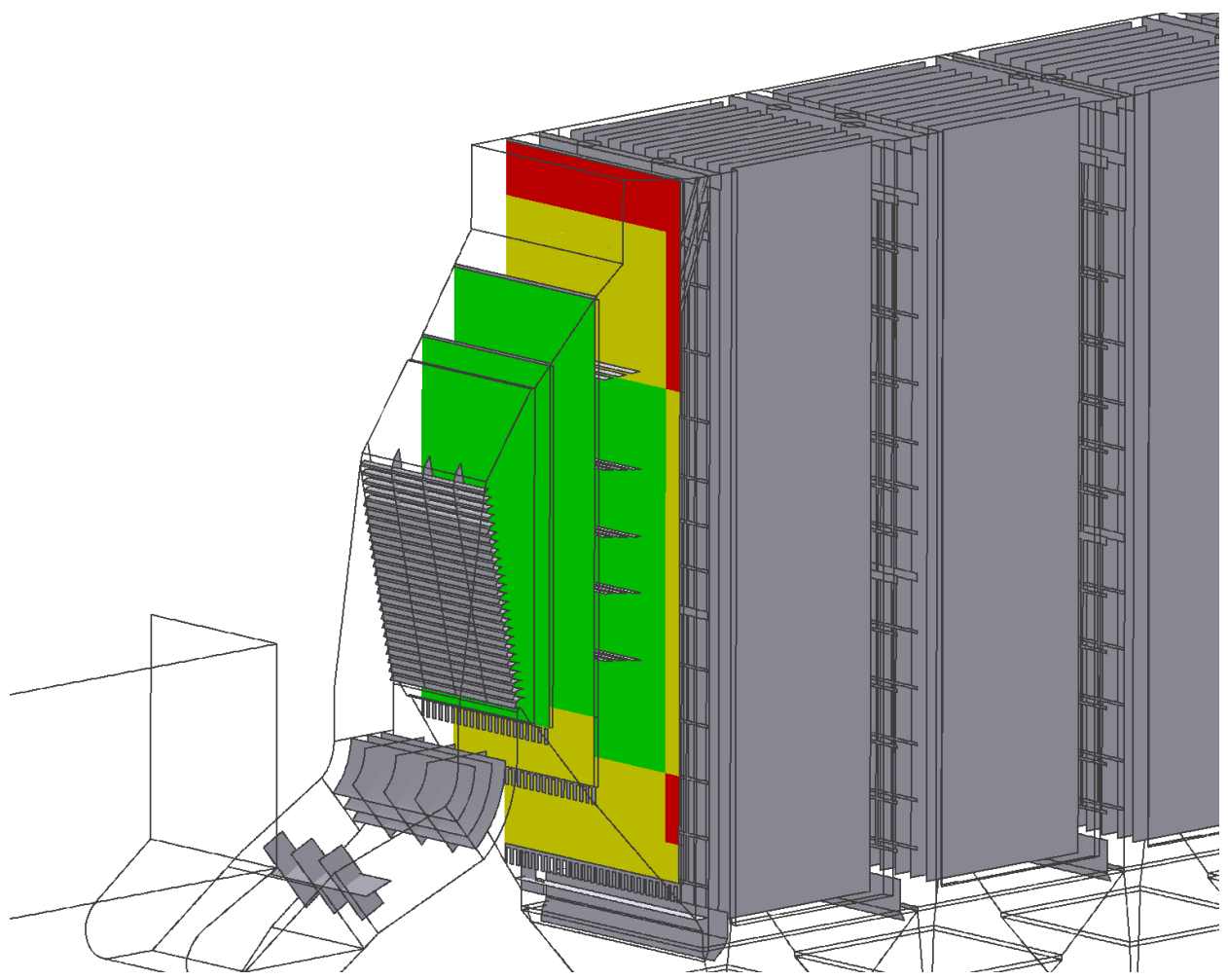

Fig. 14. Geometry of the filter and perforated plates.

On-site measurements during testing of the device found that the distribution of the stream inside the filtration chamber is not ideal because there are high values of velocities in the lower part of the filtration chamber. The CFD results show that the on-site measurements and numerical simulations agree. The result of the simulation is shown in Figure 15. It is clearly visible that the upper part of the filter is practically unused but the lower part is overloaded.

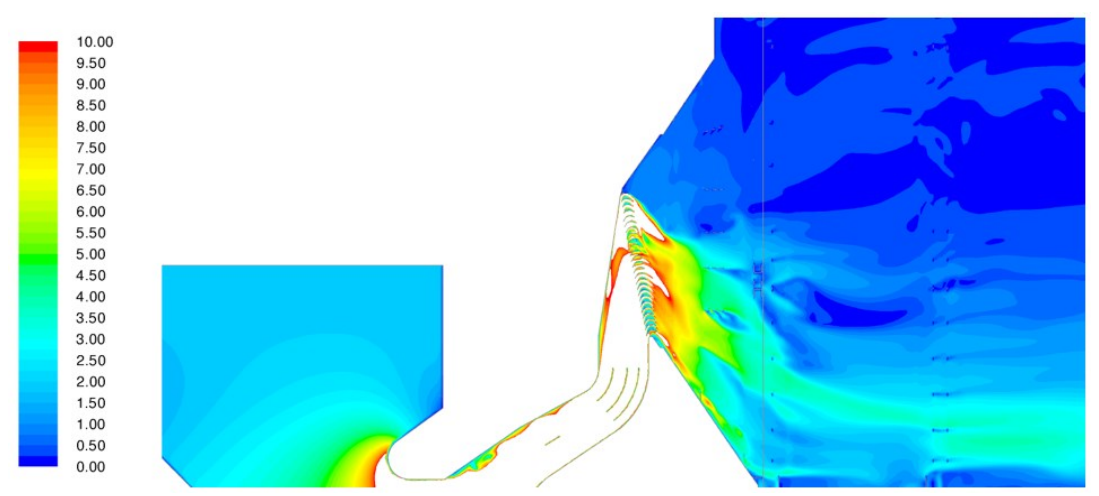

Fig. 15. Velocity magnitude contour $[\mathrm{m} / \mathrm{s}]$ of the initial variant. 
A couple of simulations were conducted and the final geometrical adjustment was chosen according to their results. The simulations showed that the perforated plates are designed well but the design of the vane wall needs to be changed. Figure 16 show that the final geometry modification considerably improves the flow distribution inside the filtration chamber. The differences compared to the original variant are the lengths and angle of the vanes.

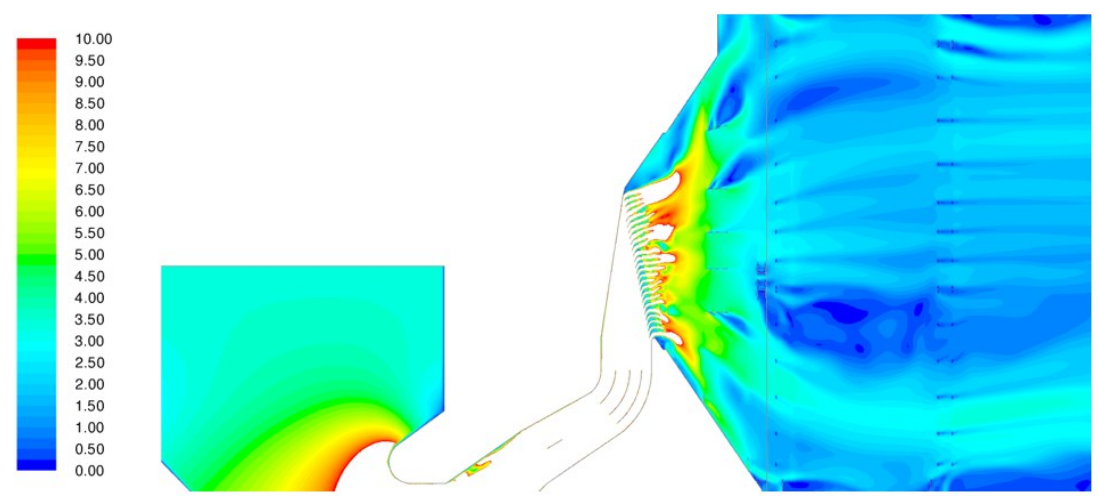

Fig. 16. Velocity magnitude contour $[\mathrm{m} / \mathrm{s}]$ of the designed variant.

The most important criterion for the final design was the distribution of the flow in front of the first section inside the filtration chamber. The other important places to check the distribution of the stream was in front of each filtration section. The results from the initial variant are shown in Figure 17. These contours prove the previous statement that the stream is mostly allocated in the lower part of the filtration chamber and so this part is overloaded. Another criterion was that the collecting hoppers should not be splashed by the stream.
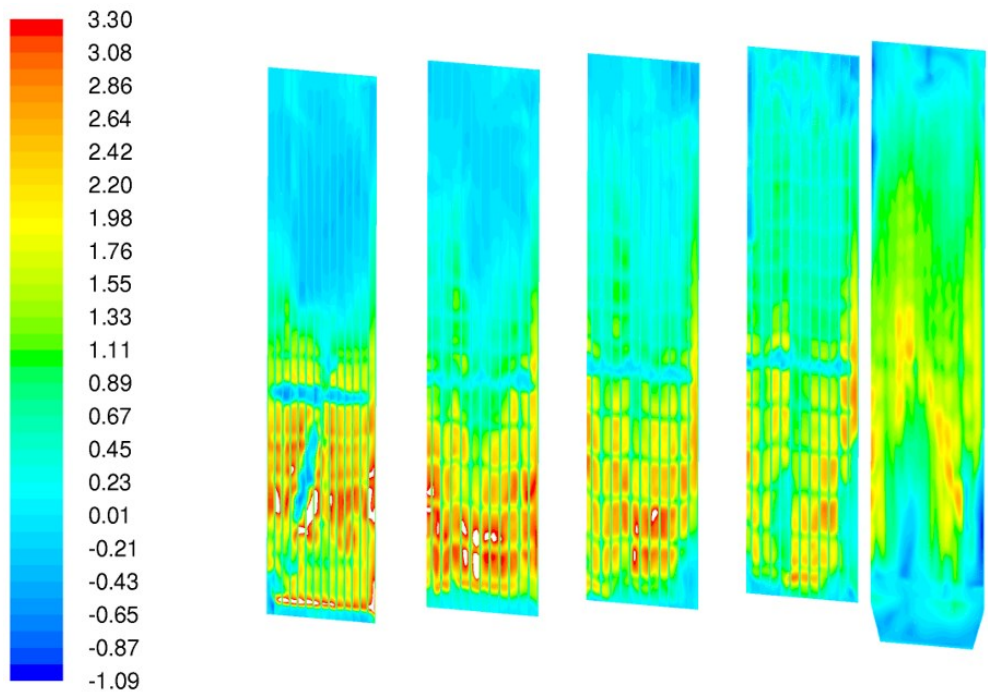

Fig. 17. Velocity magnitude contour $[\mathrm{m} / \mathrm{s}]$ of the initial variant. 
In Figure 18 are displayed the contours of the final variant. It is evident the modifications improved the whole distribution inside the filter and the collecting hoppers are not splashed by the flow.
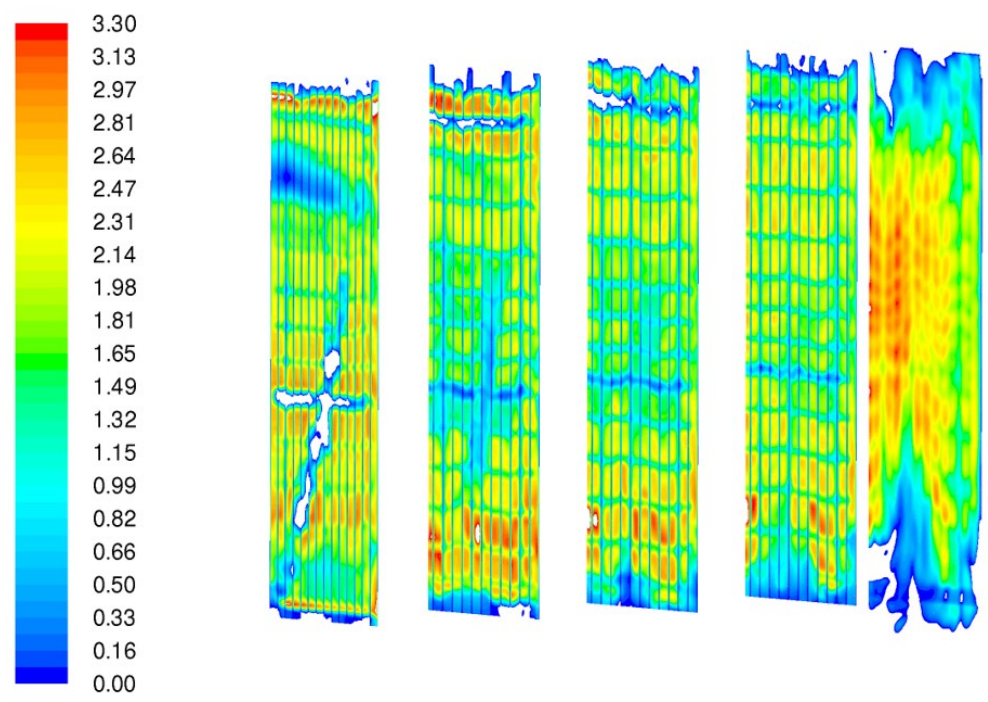

Fig. 18. Velocity magnitude contour $[\mathrm{m} / \mathrm{s}]$ of the designed variant.

The simulations were done with only one half of the filter because, as mentioned above, it is symmetrical. The mass flow of the flue gas which flows through the filter is $101.2 \mathrm{~kg} / \mathrm{s}$ and the density of the flue gas is $1.15 \mathrm{~kg} / \mathrm{m}^{3}$. These values were achieved during the on-site measurement with air so the results can be compared. The model of the ESP 3 has also been set as for the ESP 1.

Figure 19 shows the results from CFD simulation and measurement. The left picture shows the results for the original variant. The measurement and simulations were done on the left side of the filter and also on the right side. The red line with square markers is a result from the right filter part from the measurement and the blue line with markers are results from the left filter part from the measurement. The red line without markers is a result from the right filter part from the CFD simulation and the blue line without markers are results from the left filter part from the CFD simulation. On the left side of the figure is compared the initial variant. The results from CFD simulation and the experiment have very similar trends, so it is possible to say that there is quite good agreement. The right side of Figure 19 only shows the results from the simulation because the on-site measurements have not been done yet, but similar agreement is expected with the CFD simulation results. 


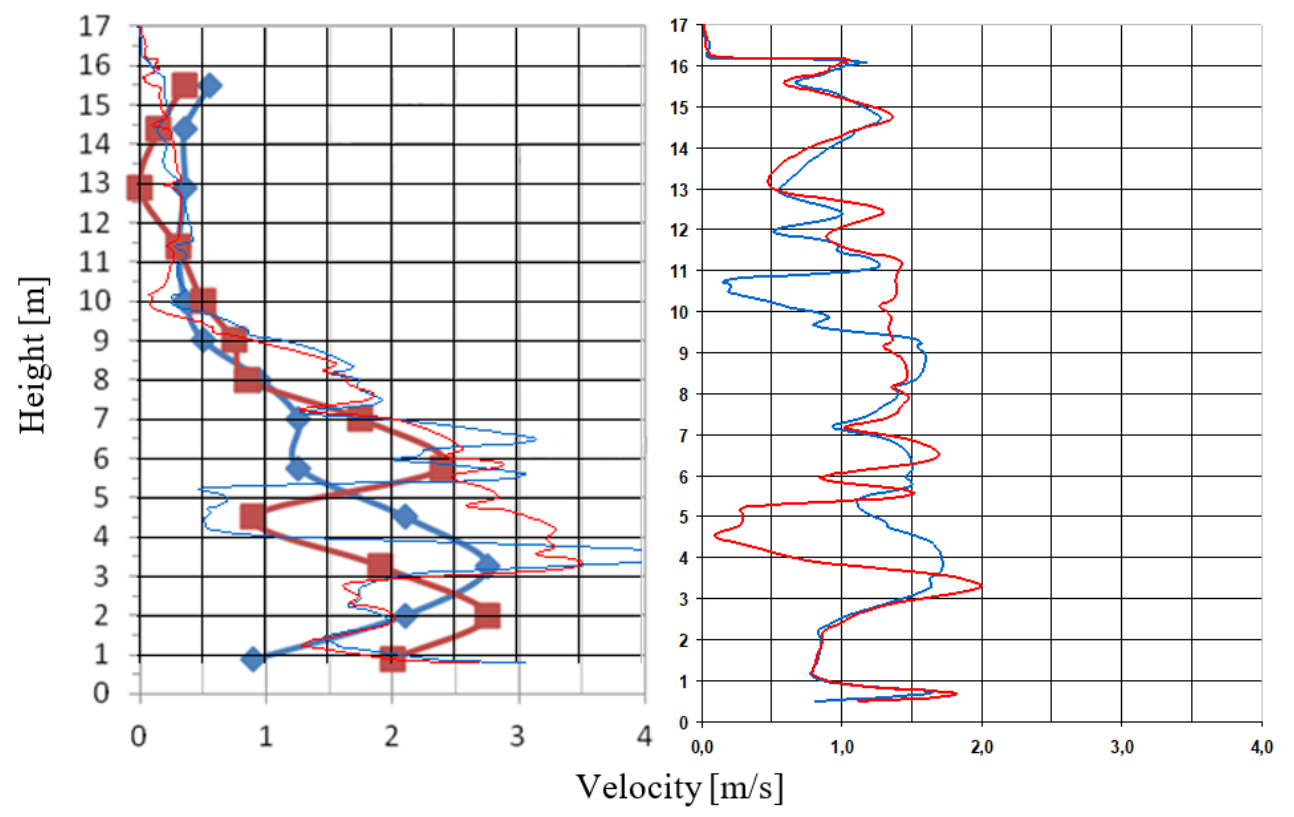

Fig. 19. Results from CFD simulation and the on-site measurement, initial variant (left), modified variant (right).

The proper distribution of the flue gas is also affected by the perforated plates at the outlet of the filter. The outlet perforated plates were also optimized to improve the filtration efficiency; this optimization was also done using CFD simulations. The outlet plates were not simplified but they were fully modelled, but the description and results of these simulations are not a part of this publication.

\section{Conclusions}

This contribution describes three numerical studies focusing on the flow in real electrostatic precipitators (ESPs). The goal of all the studies was the improvement of the flows in the ESPs.

The gas flow in an ESP is specific. The flow velocities are low enough to allow the correct functioning of the electrostatic precipitation process. The geometry of the ESP is complicated and must be simplified for CFD modelling. The dimensions are big and the computational resources and the time for the design finalization are often limited. The simulations require tens of millions cells for the cases described here. Although the validation of the simulation results by experimental measurement is difficult, expensive and not very accurate, it is very valuable. The results are often kept as trade secrets, and the publication of details of the research process is impossible in many cases.

The three cases in the article are described only briefly and simplified for the reasons mentioned above. All three ESPs were analysed in detail with many geometric modifications, simulations were run including solid pollutants, etc. Even though only a small part of the results are presented, these partial results may be practically useful because they show the following:

- CFD modelling of ESPs is very useful in the design process of the device,

- the measured and computed velocities are comparable, it is possible to obtain considerable agreement, but not exact agreement, 
- the flue gas duct in front of the ESP and the inlet part plays a significant role in the flow in the ESP chambers and its influence must be included in the design process,

- the geometry modification must be specific for each newly designed ESP as well as for reconstructions of an older one. In some cases only a small change to a construction detail change can help, but in other cases radical changes in geometry are necessary.

\section{References}

1. Electrostatic precipitator, In: Wikipedia: the free encyclopedia [online]. San Francisco (CA): Wikimedia Foundation, 2001- [cit. 2020-08-20]. Online:

https://en.wikipedia.org/wiki/Electrostatic_precipitator

2. J. F. Zhang, Z. X. Li, W. Li, Y. Gao, Q. Li, Numerical Simulation of Air Distribution Plate at the Inlet of Wet Electrostatic Precipitator. Applied Mechanics and Materials [online]. 2014, 494-495, 756-762 [cit. 2020-08-20]. DOI: 10.4028/www.scientif ic.net/AMM.494-495.756. ISSN 1662-7482. Online:

https://www.scientific.net/AMM.494-495.756

3. B. Guo, A. Yu, J. Guo, Numerical Modelling of ESP for Design Optimization. Procedia Engineering [online]. 2015, 102, 1366-1372 [cit. 2020-08-20]. DOI: 10.1016/j.proeng.2015.01.268. ISSN 18777058. Online: https://linkinghub.elsevier.com/retrieve/pii/S1877705815002878

4. S. M. E. Haque, M. G. Rasul, A. Deev, M. M. K. Khan, J. Zhou, Numerical Simulation of Turbulent Flow inside the Electrostatic Precipitator of a Power Plant. Proceedings of the 2006 WSEAS/IASME International Conference on Fluid Mechanics. Miami, 2006, 2006, 25-30.

5. M. Novák, R. Matas, J. Sedláček, Numerical simulations of flue gas flow in a first stage filter with top inlet - Modifications of the inlet chamber inserts [online]. In: 2017, s. 020026- [cit. 2020-08-20]. DOI: 10.1063/1.5004360. Online:

http://aip.scitation.org/doi/abs/10.1063/1.5004360

6. Q. F. Hou, B. Y. Guo, L. F. Li a A. B. Yu, Numerical Simulation of Gas Flow in an Electrostatic Precipitator. Seventh International Conference on CFD in the Minerals and Process Industries. Melbourne, 2009, 2009(7).

7. M. Jędrusik, Physical and numerical modelling of gas flow in electrostatic precipitator. PRZEGLAD ELEKTROTECHNICZNY [online]. 2017, 1(2), 230-233 [cit. 2020-08-20]. DOI: 10.15199/48.2017.02.50. ISSN 0033-2097. Online:

http://sigmanot.pl/publikacja-103828-2017-2.html 